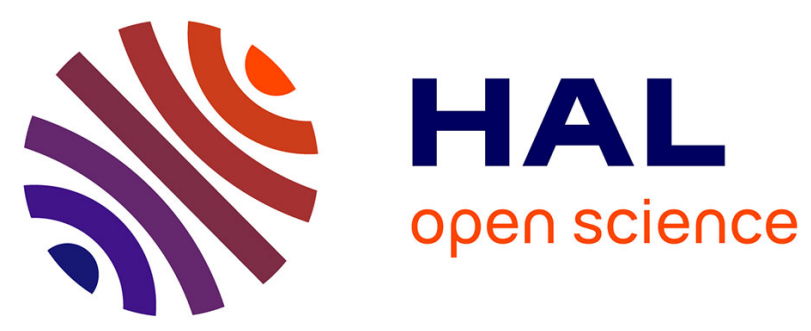

\title{
Spintronic memristors for neuromorphic circuits based on the angular variation of tunnel magnetoresistance
}

Bernard Diény, M. Mansueto, A. Chavent, S. Auffret, I. Joumard, L. Vila, R. C Sousa, L. D Buda-Prejbeanu, I. L Prejbeanu

\section{To cite this version:}

Bernard Diény, M. Mansueto, A. Chavent, S. Auffret, I. Joumard, et al.. Spintronic memristors for neuromorphic circuits based on the angular variation of tunnel magnetoresistance. Nanoscale, 2021, 13 (26), pp.11488-11496. 10.1039/D1NR00346A . hal-03360800

HAL Id: hal-03360800

https://hal.univ-grenoble-alpes.fr/hal-03360800

Submitted on 1 Oct 2021

HAL is a multi-disciplinary open access archive for the deposit and dissemination of scientific research documents, whether they are published or not. The documents may come from teaching and research institutions in France or abroad, or from public or private research centers.
L'archive ouverte pluridisciplinaire HAL, est destinée au dépôt et à la diffusion de documents scientifiques de niveau recherche, publiés ou non, émanant des établissements d'enseignement et de recherche français ou étrangers, des laboratoires publics ou privés. 


\section{Spintronic memristor for neuromorphic circuits based on the angular variation of tunnel magnetoresistance}

Received 00th January 20xx, Accepted 00th January 20xx DOI: $10.1039 / \times 0 \times x 00000 x$

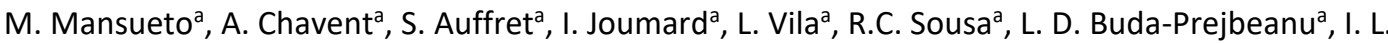 \\ Prejbeanu ${ }^{a}$ and B. Dieny ${ }^{a *}$
}

\begin{abstract}
In this study, a new type of compact magnetic memristor is demonstrated. It is based on the variation of the conductivity of a nano-sized magnetic tunnel junction as a function of the angle between the in-plane reference layer magnetization and a free layer exhibiting an isotropic in-plane coercivity. The free layer magnetization is rotated by two spin transfer torque contributions: one originating from the in-plane magnetized reference layer, the other one from an additional perpendicular polarizer integrated in the stack. Thanks to a proper tuning of the relative influence of these two torques, the magnetization of the free layer can be rotated step by step clockwise or anticlockwise in a range of angle between $0^{\circ}$ (parallel configuration) and $180^{\circ}$ (anti-parallel configuration) by sending pulses of current through the stack, of one or opposite polarity. The amplitude of the rotation steps and therefore of the conductance variations depends on the pulse amplitude and duration. In this way, we achieve monotonous variations of the resistance with the voltage polarity through the application of pulses in the ns range. We also retrieve the analytical expression of critical current density which is found to be in good agreement with the experimental results. The thermal stability of the intermediate resistance levels and the role of Joule heating are also discussed.
\end{abstract}

Kewords: Magnetic tunnel junction, tunnel margnetoresistance, memristor, exchange bias, neuromorphic, dry friction

\section{Introduction}

The exponential increase of the amount of data produced worldwide in the last decade pushes our technologies toward their limits. The total associated power consumption steadily increases every year. In this context, bio-inspired technologies seem to provide a promising solution to face these trends. In particular, neuromorphic computing can be used for fast and energy-efficient applications through the implementation of networks of artificial neurons and synapses. Some concepts of such spintronic components were proposed in the last few years, exploiting different physical mechanisms to achieve memristor functionality. Memristors are non-volatile memory devices mimicking artificial synapses, whose resistance can take several intermediate values between minimum and maximum, the resistance varying monotonously as a function of the polarity of the current pulses flowing through the device. Memristor concepts also exist based on resistive oxides ${ }^{1}$ or ferroelectric materials ${ }^{2}$. However, based on the knowledge acquired during MRAM development, the expected advantages of spintronic memristors are a better write endurance and a lower cell to cell variability than for their RRAM or ferroelectric-based counterparts. Their drawback is the lower $\mathrm{R}_{\mathrm{OFF}} / \mathrm{R}_{\mathrm{ON}}$ ratio which should be at least partially compensated by their lower cell to cell variability. A first proposed concept of spintronic memristor is based on the controlled domain wall (DW) nucleation

\footnotetext{
a. a. Univ. Grenoble Alpes, CEA, CNRS, Grenoble INP, IRIG-SPINTEC, 38000 Grenoble, France

b.*bernard.dieny@cea.fr
}

and propagation in the free layer of a magnetic tunnel junction (MTJ) ${ }^{3-5}$. The pinning and depinning of the DW at different positions in MTJ track define the intermediate resistance states. The main drawback is the reliability of the intermediate states. Moreover, the number of pinning sites, directly related to the number of intermediate resistance states, is linked to the size of the device, making it hardly scalable. Other propositions rely on the use of several binary MTJs connected in series or in parallel configurations ${ }^{6,7}$. The probabilistic switching of each individual MTJ due to Spin Transfer Torque (STT) under pulses of current of properly chosen duration and amplitude allows to realize the memristor function. In this case, the number of resistance levels strictly depends on the number of interconnected MTJs. This limits again the scalability despite the good reliability of the intermediate states. A third proposal exploits the spin orbit torque (SOT) in a SOT-MRAM cell modified to include an antiferromagnetic material constituting the heavy metal line, exchange biased with a perpendicularly magnetized storage layer ${ }^{8,9}$. A gradual switching occurs in $\mu \mathrm{m}$-sized dots under pulses of current flowing through the heavy metal line. Besides, spike-timingdependent plasticity (STDP) is observed in this device. By reducing the size of the device, the number of intermediate states reduces up to a binary behavior at few hundreds $\mathrm{nm}$ of diameter. Commonly to all those devices, the size is a limiting parameter because directly linked to the number of intermediate states.

In the present study, we realized a nano-sized memristive device whose functionalities do not depend directly on the lateral size. This, together with the other advantages of the MRAM technology, allows the creation of a scalable, reliable and easily integrable device. The 
idea is to exploit the transport properties of an in-plane magnetized tunnel junction. It is known that the conductance of such device depends on the relative orientation of the magnetizations of the two magnetic electrodes (analyzer and free layer) ${ }^{10,11}$. It varies as the cosine of the angle between the two vectors. The stabilization of the magnetization of the free layer along different in-plane directions yields the possibility to obtain intermediate resistance values between the lowest and the highest (respectively for parallel and antiparallel alignment of the two magnetizations). Concerning the writing mechanism, in order to discretely rotate the magnetization in the plane, the spin transfer torque (STT) coming from an out-of-plane polarizing layer can be exploited through the application of properly designed pulses ${ }^{12,13}$.

In a previous study, we have shown how to realize a free layer able to stabilize the magnetization along different in-plane directions ${ }^{12,13}$. Such isotropic properties can be obtained in a trilayer structure composed of two ferromagnets sandwiching a thin antiferromagnetic material. The thickness of the antiferromagnetic layer is adjusted to provide enhanced coercivity but no exchange bias at the temperature of operation (typically around $2 \mathrm{~nm}$ for IrMn at $300 \mathrm{~K})^{12}$. In such full sheet film sample, the coercive field and remanence are independent on the in-plane direction in which the magnetic field is applied. Those properties can be phenomenologically modeled with the Landau-Lifshitz-Gilbert (LLG) equation including a dry friction-like term. The addition of this dissipative term led to a good agreement with the experimental results. Moreover, the integration of such layer in a nano-sized pillar preserves its isotropic properties ${ }^{13}$ as confirmed by rotating field experiment and measurement of the tunnel magnetoresistance (TMR) variation versus in-plane angle.

To achieve a memristor behavior which requires a monotonous variation of the resistance versus current pulse polarity, it is important to limit the angular excursion of the free layer magnetization between $0^{\circ}$ and $180^{\circ}$ corresponding respectively to the parallel and antiparallel magnetic configuration. In our earlier study ${ }^{12}$, we showed that this could be achieved by the application of a static transverse field of suitable amplitude acting on the free layer magnetization. This field is applied in-plane perpendicular to the reference layer magnetization. However, applying such field is not very convenient in practical devices which motivates for instance the search for field free implementation in the context of SOT-MRAM ${ }^{14}$ To circumvent the difficulties associated with application of a static field, we show in this study that, by properly adjusting the two STT relative contributions (from the perpendicular polarizer and the inplane reference layer), it is possible to gradually rotate the magnetization of the free layer clockwise or counterclockwise depending on the pulse polarity, within a $0^{\circ}-180^{\circ}$ excursion, thus achieving memristor functionality.

In the framework of our model, we distinguish different working regions for our device as a function of the two STT contributions. Guided by the simulations, we realize a nano-sized device exhibiting the expected memristive properties.

\section{Model}

The proposed concept is actually an extension of a previously studied memory device investigated for the realization of sub-ns binary switching thanks to the phenomenon of precessional switching induced by a perpendicular polarizer on an in-plane magnetized free layer ${ }^{15-20}$. This device was sometime called Orthogonal Spin Transfer Torque MRAM (OST-MRAM) ${ }^{16}$. In the present study, the original device is modified by introducing an isotropically coercive free layer instead of a bistable free layer with uniaxial anisotropy. In fact, the idea is to exploit the STT coming from the perpendicular polarizer to pull the magnetization of the free layer out-of-plane and make it rotate around the demagnetizing field. By properly adjusting the pulse amplitude and duration, the magnetization can be switched by $180^{\circ}$. Besides, it was shown that by playing with the relative contributions of the two STT (from perpendicular polarizer and from planar reference layer), one can achieve $180^{\circ}$ switching independently of the pulse duration which makes the concept much easier to implement in practical devices ${ }^{17,19}$. We will show that the same is true for the present memristor device.

Our model based on a modified LLG equation with the addition of a dissipative dry friction term ${ }^{12}$ is used here to investigate the free layer magnetization $(\boldsymbol{M})$ dynamics in such device:

$\frac{\partial \boldsymbol{M}}{\partial t}=-\gamma\left(\boldsymbol{M} \times \mu_{0} \boldsymbol{H}_{\text {eff }}\right)+\frac{\alpha_{G}}{M_{S}}\left(\boldsymbol{M} \times \frac{\partial \boldsymbol{M}}{\partial t}\right)+\beta M_{S} \frac{\boldsymbol{M} \times \frac{\partial \boldsymbol{M}}{\partial t}}{\left|\boldsymbol{M} \times \frac{\partial M}{\partial t}\right|}$

where $\gamma$ is the gyromagnetic ratio, $\alpha_{G}$ the Gilbert damping, $M_{S}$ the saturation magnetization and $\beta$ the dry friction parameter. In this equation, the first term describes the magnetization precession around the effective field, the second term represents the Gilbert damping dissipation and the third term the dry friction term associated with the exchange like coupling with the thin antiferromagnetic layer ${ }^{12,13}$. From the magnetization dynamics, the resistance variation can be straightforwardly derived from the expression of the conductance of a magnetic tunnel junction ${ }^{10}$ :

$G=\frac{1}{R}=G_{90^{\circ}}+\frac{\Delta G}{2} \frac{\boldsymbol{M} \cdot \boldsymbol{M}_{R}}{M_{s} \cdot M_{R s}}$

Where $G_{90^{\circ}}$ is the junction conductance in the configuration when the free layer magnetization is orthogonal to that of the reference layer (also called analyzer), $\Delta G$ is the absolute magnetoconductance defined as the variation of conductance between the parallel magnetic configuration (free layer magnetization parallel to that of the reference layer, high conductance state) and antiparallel configuration (low conductance state). $\Delta G$ is related to the usual tunnel magnetoresistance $T M R=\frac{\Delta R}{R_{P}} \quad(\Delta R \quad$ absolute magnetoresistance and $R_{P}$ resistance in parallel configuration) by $\Delta G=\frac{T M R}{R_{P}(1+T M R)}$. In (2), $\boldsymbol{M}_{\boldsymbol{R}}$ is the reference layer vector magnetization and $M_{R S}$ its amplitude.

We first analyze the contribution of torque coming from the in-plane analyzer (i.e. reference layer) by including in the equation the torque $\boldsymbol{T}_{\boldsymbol{a n}}=\gamma a_{a n / /} V \boldsymbol{M} \times(\boldsymbol{M} \times \boldsymbol{L})$ where $L=\left(\begin{array}{lll}1 & 0 & 0\end{array}\right), V$ is the applied voltage and $\boldsymbol{a}_{\boldsymbol{a n} / /}$ is the Slonczewski prefactor. This last can be expressed as $a_{a n / /}=\frac{\hbar}{2 e} \frac{\eta_{a n}}{\boldsymbol{l} \boldsymbol{M}_{\boldsymbol{s}} \boldsymbol{R} \times \boldsymbol{A}}$, where $l$ is the thickness of the free layer, $R \times A$ the resistance $\mathrm{x}$ area product and $\eta_{a n}$ the spin torque efficiency. All the following results are obtained for a layer of cylindrical shape, $100 \mathrm{~nm}$ in diameter, and at $T=0 K$. Looking for in- 
plane $\left(\mathrm{M}_{\mathrm{z}}=0\right)$ stationary solutions $\left(\frac{\partial M}{\partial t}=\mathbf{0}\right)$, we find that the threshold current density can be expressed as

$J_{a n}=\frac{\beta M_{S} l 2 e}{\gamma \hbar \eta_{a n}}$

In a similar way, considering the STT from the perpendicular polarizer only: $\quad \boldsymbol{T}_{\text {perp }}=\gamma a_{\text {perp } / /} V \boldsymbol{M} \times(\boldsymbol{M} \times \boldsymbol{P})$ with $\mathbf{P}=\left(\begin{array}{lll}0 & 0 & 1\end{array}\right)$, it is possible to compute the critical current required to trigger the outof-plane precession (OPP) :

$J_{\text {perp }}=\frac{\beta M_{S} l 2 e}{\gamma \hbar \eta_{\text {perp }}}$

If now both torques are inserted in the equation, the critical current density for which in-plane static solutions become unstable writes:

$J_{\text {double }}=\frac{\beta M_{S} l 2 e}{\gamma \hbar} \frac{1}{\sqrt{\eta_{\text {perp }}^{2}+\eta_{a n^{2}}}}$

In Fig. 1, the three critical lines are drawn with respect to the spin torque efficiency of the perpendicular polarizer $\eta_{\text {perp }}$ for a fixed value of $\eta_{a n}=0.3$ (obtained from the measured TMR value of the device with the formula $\left.\eta_{a n}=\frac{\sqrt{T M R(T M R+2)}}{2(T M R+1)}{ }^{21}\right)$.

Considering a generic initial configuration of the magnetization of the free layer, the dotted line $J_{a n}$ represents the limit of stability of such state. Below this threshold, there is the stability region where the magnetization is at equilibrium whatever its in-plane direction, while, above it, the magnetization becomes unstable. The dashed line $J_{\text {perp }}$ is the critical line for the excitation of out-of-plane precession of the magnetization (OPP). Above this line only OPP can occur. The green dashed dotted line $J_{\text {double }}$ represents the correction to the stability line $J_{a n}$ due to the other STT contribution originating from the perpendicular polarizer

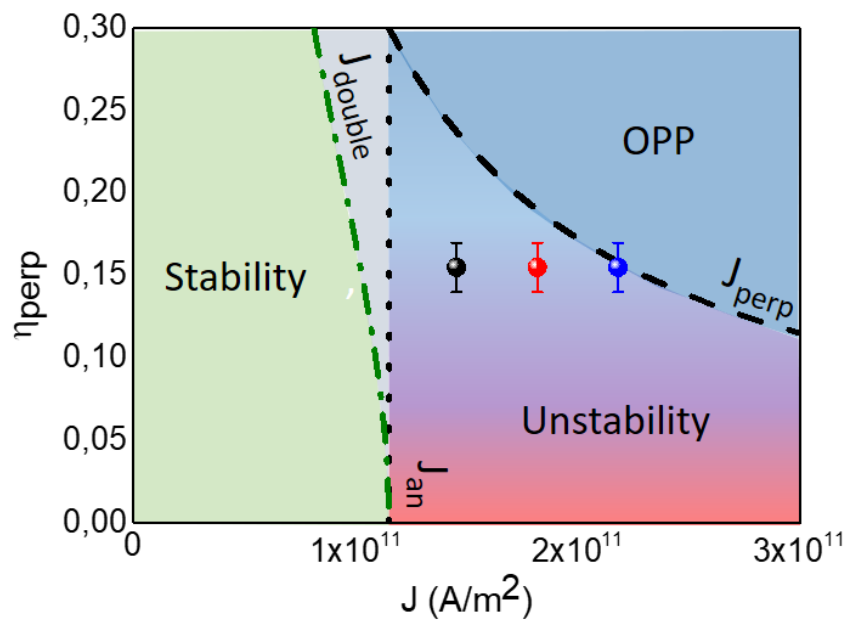

Fig.1 Analytical diagram of the working regions for a device with $\eta_{a n}=0.3$. The experimental points in black, red and blue correspond to an applied voltage respectively of $0.8 \mathrm{~V}, 1 \mathrm{~V}$ and $1.2 \mathrm{~V}$. The parameters used for all the simulations presented in this work are: $M_{s}=10^{6} \mathrm{~A} / \mathrm{m}, \alpha_{G}=0.04, R \times A=10 \Omega \mu \mathrm{m}^{2}, l=4 \mathrm{~nm}, \beta=5 \mathrm{x}$ $10^{8} \mathrm{rad} / \mathrm{s}, \mathrm{K}_{\mathrm{u}}=0 \mathrm{~J} / \mathrm{m}^{3}, \mathrm{~N}=\left(\begin{array}{ll}0.050 .050 .899\end{array}\right)$.
As for the precessional switching mechanism, a magnetic stack comprising two similar tunnel junctions would be ideal to fully exploit the OPP region. In this case, close to the top part of Fig. 1, the OPP would start as soon as the magnetization is no more in the stability region because of the dominant effect of the STT contribution from the perpendicular polarizer. However, as we will see further, this would not allow to keep the angular excursion of the free layer magnetization between $0^{\circ}$ and $180^{\circ}$ and therefore to achieve the required monotonous variation of resistance for a given pulse polarity.

In the unstablity region, where the two STT contributions are competing, it is not possible to exactly define analytically the magnetization dynamics. For values of $\eta_{\text {perp }}$ close to zero, the STT torque from the analyzer is dominating, leading to a binary switching of the free layer. In this case, the addition of the dry friction term blocks the stochastic behavior at the beginning of the switching mechanism, making the magnetization switching almost impossible at low current densities (blue line in Fig. 2a). By increasing the torque originating from the perpendicular polarizer, the sum of the two contributions leads to a binary switching but in a time significantly shorter than in the previous case (orange line in Fig.2a). This is due to the initial torque impulse induced by the perpendicular polarizer which pulls the free layer magnetization away from the analyzer magnetization direction. In this region, the torque $T_{a n}$ favors a switching towards parallel or antiparallel configuration through a precessional trajectory around the demagnetizing field under the influence of $T_{\text {perp }}$. For higher values of $\eta_{\text {perp }}$, the magnetization starts to precess because of the dominant influence of the perpendicular STT with a trajectory distorted by the STT term from the in-plane analyzer (green line in Fig. 2a).

a)

b)
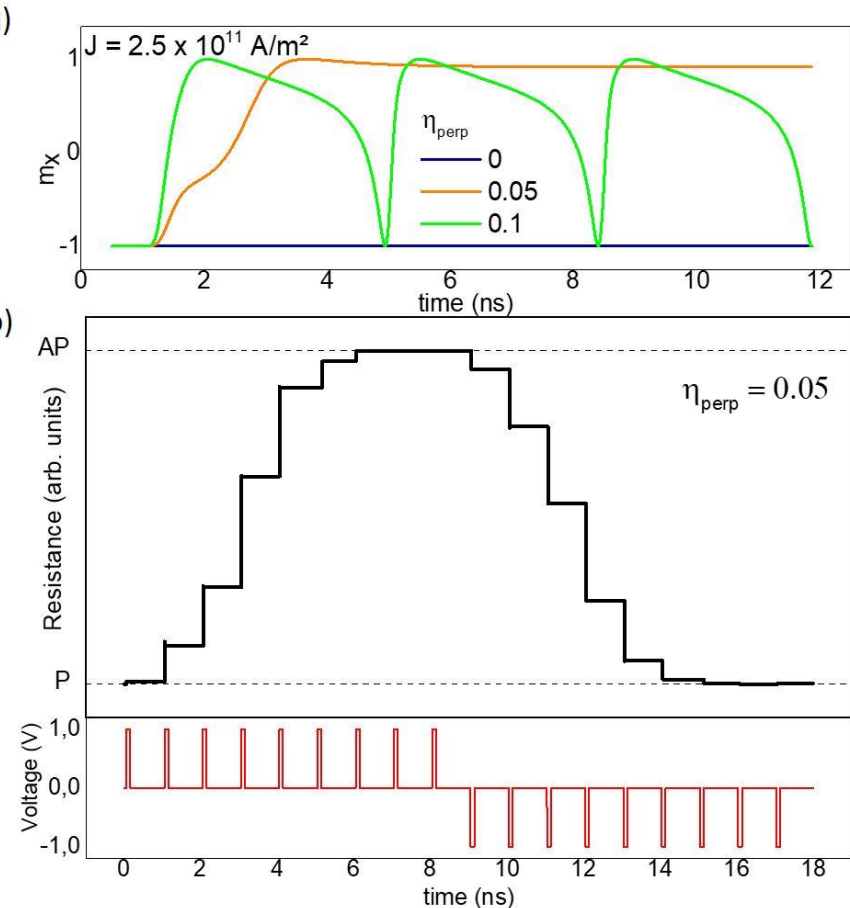

Fig.2 a) Magnetization dynamics under STT in the unstability region for different values of $\eta_{\text {perp }}$ and for current density $\mathrm{J}=2.5 \times 10^{11}$ $\mathrm{A} / \mathrm{m}^{2}$. b Resistance variation (top panel) induced by a train of pulses 
as in the bottom panel (amplitude of $1 \mathrm{~V}$, duration $1 \mathrm{~ns}$ ) for $\eta_{\text {perp }}=$ 0.05 and zero applied field.

For the final purpose of realizing a memristive behavior, the two regions of OPP and unstability could be exploited. The first case has been already simulated in ref 12 leading to a memristive behavior with pulses in the ps-range. Despite this, the monotonicity of the resistance variation with the polarity of the voltage can be achieved only with the application of an in-plane transverse field which limits the angular excursion of the free layer magnetization only in a half plane (between $0^{\circ}$ and $180^{\circ}$ ). Moreover, because of the very short pulse width, due to the relatively high frequency of oscillations, the write margin in terms of pulse duration and amplitude is very small (as for the precessional switching case). For these reasons, working in the unstability region and adjusting the relative contributions of the two torques to limit the angular excursion of the free layer magnetization in a half plane seems to be a better option for this application. In particular, the fast binary switching, as the orange line in Fig. 2a, represents an ideal switching mechanism for our case. Indeed, it is then possible to gradually rotate the magnetization step by step under pulse of currents (See Fig.2b) while limiting the magnetization excursion between the $P$ and AP states. This ensures the monotonicity of the resistance variation for a given polarity of the voltage even without the application of an in-plane field. Because of those advantages, we chose to fabricate a device designed to operate in the unstability region of Fig. 1.

\section{Magnetic stack}

To exploit the effects of the combined STT in the region described above with a torque efficiency from the perpendicular polarizer being about one sixth of the torque efficiency from the in-plane analyzer (corresponding to the orange curve in Fig. 2a), the isotropically coercive free layer is sandwiched between a Cu metallic spacer separating it from the perpendicular polarizer and a tunnel barrier separating it from the in-plane analyzer (i.e. reference layer) as depicted in Fig. 3. Indeed, it is known that the spin-transfer torque efficiency in metallic spin-valves is significantly lower than in magnetic tunnel junctions ${ }^{16}$. This allows the device to operate in the unstablity region of the diagram in Fig. 1 with $\eta_{\text {perp }}$ significantly lower than $\eta_{a n}$ and, at the same time, have a reading mechanism based on the TMR of the top junction.

In the magnetic stack, the polarizer and the analyzer are implemented as synthetic antiferromagnets with an RKKY antiferromagnetic coupling through a thin Ru spacer 22-24. In the polarizer, the perpendicular magnetization is induced by the $(\mathrm{Co} / \mathrm{Pt})$ multilayers of composition $[\mathrm{Co}(0.5) / \mathrm{Pt}(0.25)]_{6}$ and $[\mathrm{Co}(0.5) / \mathrm{Pt}(0.25)]_{3}$ (in parenthesis, the thicknesses are in $\left.\mathrm{nm}\right)^{25}$. The reference is a $\mathrm{Co}(1)$ layer followed by the spacer $\mathrm{Cu}(3)$ with a lamination of $\mathrm{Cu}(0.2)$ to enhance the spin-polarization thanks to a shortening of the spin-diffusion length ${ }^{15}$. The isotropic free layer $\mathrm{Py}(1) / \mathrm{IrMn}(2.1) / \mathrm{Py}(1)$ is inserted between a layer of $\mathrm{Co}(0.6)$ and a layer of $\mathrm{FeCoB}(1.6)$ to optimize the STT coming from both sides and the TMR of the top junction. This last is based on an $\mathrm{MgO}(1.2)$ tunnel barrier with an RxA around $6 \Omega \cdot \mu \mathrm{m}^{2}$. As in conventional STT-MRAM the $\mathrm{MgO}$ barrier is surrounded by two FeCoB electrodes themselves separated from the rest of the stack by two thin Ta layers. These Ta laminations aim at absorbing the B from the $\mathrm{FeCoB}$ electrodes upon annealing of the structure and insuring a structural transition between the $\mathrm{FeCoB} / \mathrm{MgO} / \mathrm{FeCoB}$ body centered cubic (bcc) part of the stack and the bottom and top parts which have a face centered cubic (fcc) structure ${ }^{26}$. The top SAF is based on a $\mathrm{Co}(1)$ and $\mathrm{Co}(2.5) / \mathrm{IrMn}(7)$ structure with a $\mathrm{FeCoB}(1.2)$ in contact with the MgO. The overall stack may look complex. However, its structure as well as the materials used in the different layers comprised in the stack are very similar to those used in STT-MRAM cells as well as in magnetoresistive heads implemented in hard disk drives ${ }^{27}$. Therefore, this type of stack is manufacturable without major difficulties using state-of-the art fabrication tools

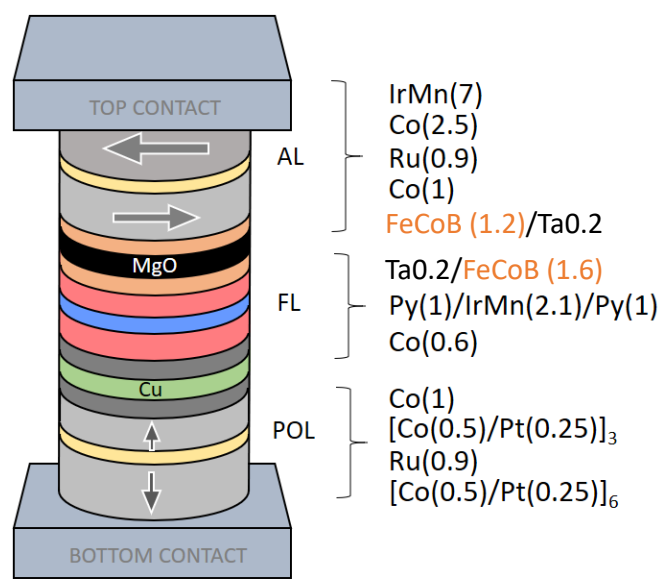

Fig.3 Schematic of the memristive device with the detailed composition

The devices are fabricated in arrays of pillars with diameters between $80 \mathrm{~nm}$ and $200 \mathrm{~nm}$. After nanofabrication, the isotropic properties of the free layer have been confirmed ${ }^{13}$.

\section{Single pulse experiments}

The first experiments aimed to find the pulse characteristics (amplitude and duration) for which the STT yields a discrete rotation of the free layer magnetization and consequently discrete variation of resistance value. To do this, the device was first set in the parallel state through the application of a strong in-plane field. Then, a single pulse was applied followed by the read out of the final resistance state. The device was reset in the P state before each pulse. This procedure was repeated for different pulse amplitudes and durations similarly to a switching probability experiment on a binary device. In this case, instead of the switching probability, we expect to see the actual resistance variation dependence on the pulse parameters. The resulting experimental points (each averaged over 10 measurements) are shown in Fig. 4a for a junction with a nominal diameter of $100 \mathrm{~nm}$ and a TMR of $30 \%$. 
a)

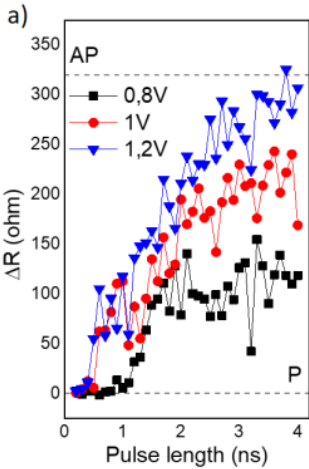

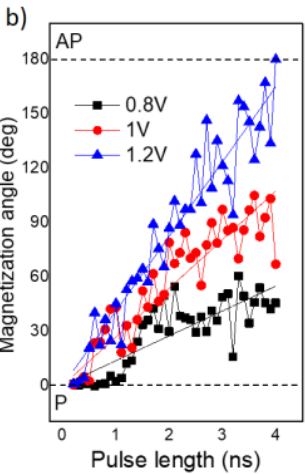

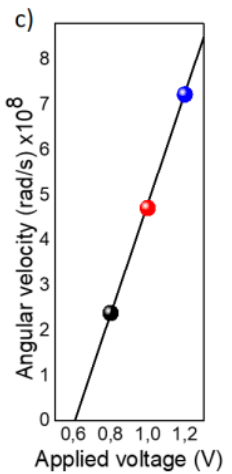

Fig.4 a) Resistance variation with the pulse duration for different pulse amplitudes. b) In-plane angle of the magnetization varying with the pulse duration as in a). The black, red and blue fitting lines correspond respectively to angular velocity of the magnetization. c) Linear fitting of the angular velocity variation with the pulse amplitude.

The magnetization angle is directly extracted from the measurement through the cosine variation of the conductance ${ }^{11}$ and shown in Fig. $4 \mathrm{~b}$. As expected, a monotonicity of the resistance variation is obtained with respect to both the pulse amplitude and duration. For each amplitude of the voltage, a threshold pulse length separates a region in which the magnetization is not moving from a region in which discrete rotations are obtained. Those threshold values are decreasing with the increase of the voltage amplitude, as expected. The experimental points are fitted with a line that represents an average behavior assuming that the magnetization angle varies linearly with time (constant angular velocity). We note that the oscillation frequencies extracted turn out to be significantly lower than the $\mathrm{GHz}$ range. This is likely the result of the important increase of the Gilbert damping already observed in FM/AF bilayers ${ }^{29,30}$ as well as FM/AF/FM trilayers ${ }^{31}$ and expected from the expression

$\omega_{M}=-\frac{\gamma a_{p e r p / / V}}{\alpha_{G}}-\frac{\beta}{\alpha_{G} \sin \left(\theta_{M}\right)} \frac{\omega_{M}}{\left|\omega_{M}\right|}$

from ref ${ }^{12}$, where $\theta_{M}$ is the magnetization polar angle. The linear behavior of this last equation with respect to the applied voltage can be used to fit the experimental points as in Fig. 4c. From this fit, the extracted threshold voltage for the triggering of the OPP is around $0.6 \mathrm{~V}$. From this value, that corresponds to the critical line $J_{\text {perp }}$ in eq. 4 , one can estimate a value of $\eta_{\text {perp }} \approx 0.15 \pm 0.02$ that leads, through eq. 6 , to a value of $\alpha_{G} \approx 0.07 \pm 0.01$. The experimental points can be compared to the analytical diagram in Fig. 1 . The comparison indicates that the device operates indeed in the unstability region as expected. Note that, in order to achieve the limited angular excursion of the magnetization (as in the orange curve in Fig. 2a), a ratio $\eta_{a n} / \eta_{\text {perp }} \approx 2$ seems to be sufficient experimentally.

Despite the fact that the values of $\eta_{\text {perp }}$ and $\alpha_{G}$ perfectly match the expectations, they can be affected by an error coming from the fact that the equations used to extract them are valid in the OPP region and in the long pulses approximation while the experimental points are taken in the unstability region where no analytical expressions can be computed.

Also, the importance of the STT coming from the perpendicular polarizer is confirmed by the fact that, in devices as in Fig. 3 but without perpendicular polarizer, it is observed that no switching occurs for pulse parameters as in Fig. 4 a, exactly as predicted by the blue line in Fig. 2a.

\section{Multiple pulses experiments}

In the following experiment, a train of pulses was sent to the device to observe the evolution of its resistance. The device was set in the $P$ state only at the beginning and not between two consecutive pulses as in the previous experiment. The pulses were designed in a way to not switch completely the magnetization but to rotate it so as to reach several intermediate resistance values. For this reason, the voltage amplitude was set to $0.9 \mathrm{~V}$ with a varying pulse duration for the results shown in the following. An important parameter chosen for this experiment was the delay between two consecutive pulses that was set to $1 \mathrm{~s}$ to get an idea of the thermal stability of the intermediate resistance states (discussed in the next section). The resistance variation to a series of 50 pulses of positive polarity is shown in Fig. 5a for different pulse durations. The magnetization, that is not much affected by the 1 ns pulses, gradually rotates towards the AP state proportionally to the pulse duration. The resistance is then saturating to the AP state confirming that the effect of the double STT acts as in the unstable region of Fig. 1 and not as in OPP (for which the resistance is supposed to oscillate with the same polarity of the voltage). In both insets of Fig. $5 a$, it can be observed that the intermediate resistance states turn out to be thermally stable along the $1 \mathrm{~s}$ between the two pulses confirming the possibility to stabilize the magnetization along different in-plane directions at least during a 1s period. One observes that although the overall trend of the resistance variation under a train of pulses of same polarity is monotonous, the individual resistance jumps are not always varying in a monotonous way (as in the two insets of Fig. 5a). A random telegraph type of noise in the resistance variation seems to be superposed to the general trend. For this reason, the number of pulses needed to completely switch from the P to the AP state is larger than what would be predicted from Fig. 4a (for which an average over 10 measurements reduces the random effect). This effect, that will be discussed in the next paragraph, is not dependent on the voltage polarity as shown in Fig. 5b. Even if the STT effect is visible in the monotonicity of the average resistance with the polarity of the voltage, the presence of random resistance jumps associated with voltage pulses persists even in the P or AP states where the STT from the in-plane analyzer is supposed to be zero (but not the one from the perpendicular polarizer). We emphasize that what we note as $P$ and AP states here are not fully saturated $P$ and AP states but rather remanent state of the free layer magnetization along the $P$ and AP directions. We know already from earlier sheet film studies ${ }^{12}$ that the hysteresis loops of the free layer are not square. The remanence is about $80 \%$ of the saturation magnetization (Fig. $4 \mathrm{~b}$ in ref 12). At the microscopic scale, due to the randomness induced by the interfacial coupling between the ferromagnetic and antiferromagnetic layer, the magnetization of the free layer is viewed as being distributed in a cone around an average direction. This explains why the resistance jumps around the nominally AP state can reach higher resistance values than those represented by the dotted lines in Fig.5a and $b$ which correspond to the resistance value in the 
AP remanent state and not AP saturated state. The same reasoning applies around the $P$ state for resistance jumps reaching resistance values below that in the remanent $P$ state.

Note also that the P-AP and AP-P transitions are asymmetric because of the residual dipolar field from the uncompensated out-of-plane SAF and/or the in-plane one that favors precession only towards one direction.
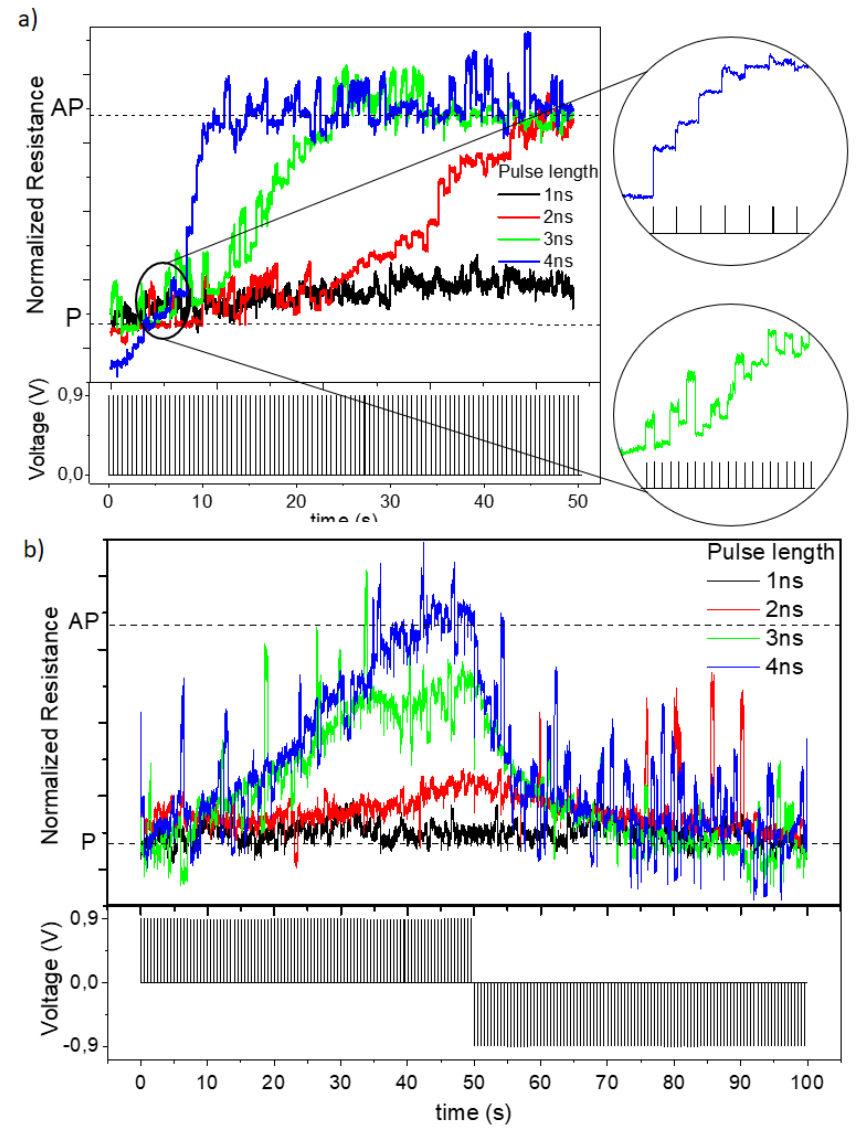

Fig.5 a) Resistance variation due to a train of pulses of $0.9 \mathrm{~V}$ with varying pulse durations and $1 \mathrm{~s}$ delay between two consecutive pulses. In the zooms, the blue curve shows an almost monotonous behavior while the green curve exhibits an average monotonous trend of the resistance variation superposed with random jumps. b) Resistance variation under a train of pulses of both polarities. These measurements were performed on two different MTJs of nominal diameter $100 \mathrm{~nm}$ exhibiting a TMR of $30 \%$.

\section{Temperature effect}

The presence of a thin IrMn layer in the free layer, with a blocking temperature of the order of $100^{\circ} \mathrm{C}$, makes this device sensitive to temperature. For this reason, the effect of temperature was investigated in terms of stability of the intermediate resistance states and Joule heating induced by the voltage pulses.

A first glimpse of the thermal stability of the resistance levels reached through STT is shown in the insets of Fig. 5a. An example of resistance distribution associated with such curve is shown in Fig. 6a. The distribution of intermediate states depends on the pulse amplitude and duration since the angular step that the storage layer magnetization makes at each pulse depends on these parameters. The distribution shown in Fig.6a was derived from the resistance variation shown in Fig.5a for pulse 3 ns long. An almost continuous range of resistances is achieved between the $\mathrm{P}$ and AP states. To analyse the stability of single resistance levels, only few levels were selected as in Fig. $6 \mathrm{~b}$. The selection criteria in order to make them distinguishable considers that the distance between two peaks has to be greater than the largest FWHM of the two distributions. The distribution of such resistances show a FWHM below $3 \Omega$, meaning a resistance variation below $0.1 \%$ in a time range of $1 \mathrm{~s}$.

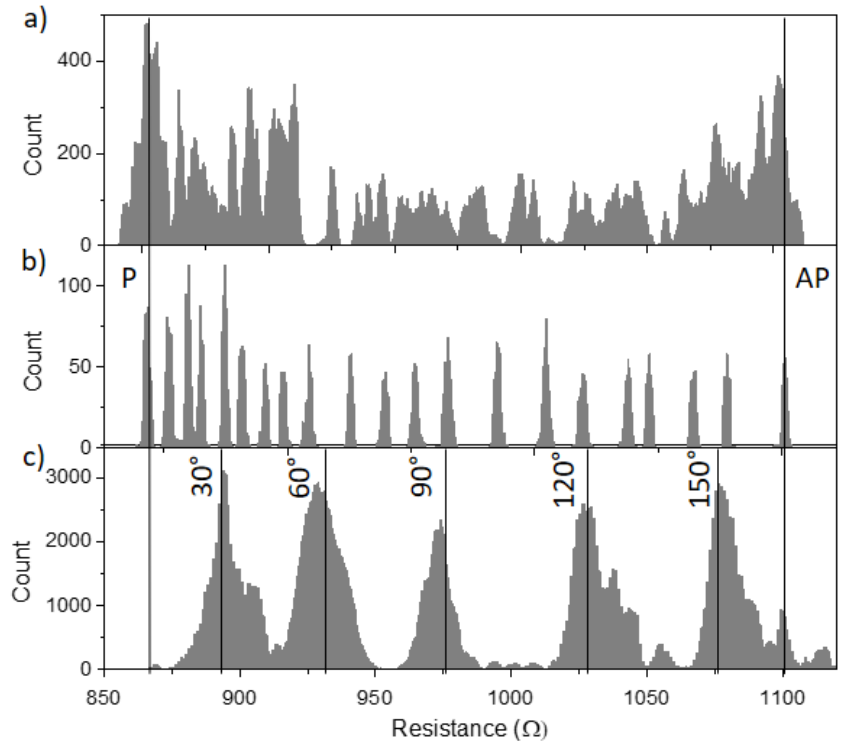

Fig.6 a) Resistance distribution in a multiple pulse experiment as in Fig. 5a. b) Resistance distribution for few distinguishable levels. c) Resistance distribution for intermediate resistance values induced by field saturation at different in-plane angles for a $3 \min$ timescale. The measurements shown in this figure were performed on a 100nm MTJ with a TMR around $26 \%$.

To determine the thermal stability of the resistance states at RT, the magnetization was saturated, through the application of a strong inplane field, in different directions and the resistance was measured for 3 minutes. As can be seen from Fig. $6 c$, the FWHM is around $17 \Omega$ with a resistance variation below $2 \%$. The results suggest that, for a device with TMR $100 \%$ and same $R \times A$, about 50 intermediate states could be used for applications in which the read/write access would occur at typical frequency of a few $\mathrm{Hz}$. Moreover, this confirms that the resistance jumps, observed in the previous experiment and that occur during the pulses, are mainly due to Joule heating and correspond to thermally activated switching of small domains in the free layer. To further investigate this, we tried to separate the effects of STT and Joule heating on the resistance variation. For each polarity of the voltage, to minimize the role of the STT compared to that of Joule heating, we set the system close to the P or AP state. This strongly reduces the STT from the in-plane analyzer but not that of the perpendicular polarizer. A statistical analysis of the resistance jumps due to a train of pulses in the saturated AP state is shown in Fig. 7a for different pulse lengths in a device of $150 \mathrm{~nm}$ of diameter. Interestingly, the distributions of jumps turn out to be peaked 
around an average value corresponding to a small fraction of the normalized TMR. This means that, when the magnetization is in the AP state, the temperature effect is to switch the magnetization of a relatively small part of the free layer with a certain characteristic size. Moreover, this area is observed to increase with the pulse length which can be ascribed to a more significant heating of the device with longer pulses (Fig. 7a). From the knowledge of the amplitude of the resistance jumps (plotted as horizontal scale in Fig.7a), one can derive the size of the switching domains, the distribution of which is plotted in Fig.7b. The Joule heating activates the switching of small portions of the free layer as it would do in a granular layer.

a)

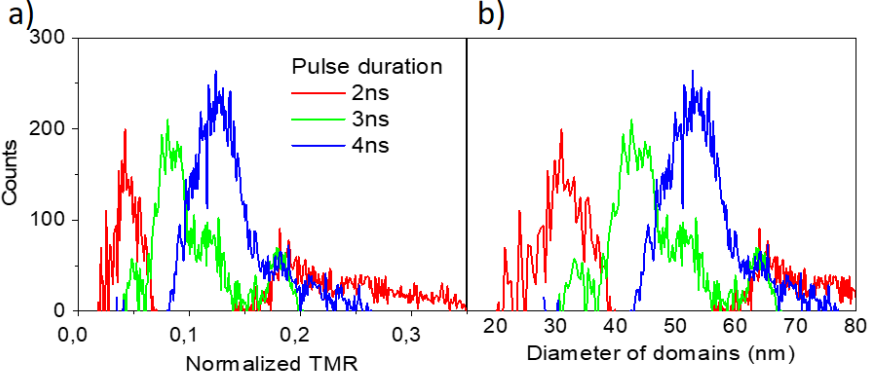

c)

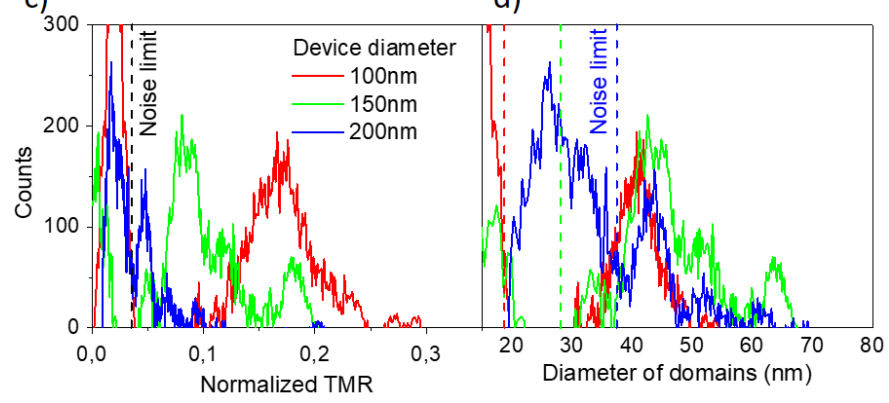

Fig.7 a) Resistance jumps distributions for different pulse lengths in a device of diameter $150 \mathrm{~nm}$. b) Resistance jumps distributions as in a) expressed versus diameter of the switching domains. c) Resistance jumps distributions for different device diameters and pulse length of $3 \mathrm{~ns}$. d) Resistance jumps distributions as in c) normalized with respect to the corresponding diameters.

To further investigate this, the same analysis was performed on devices with different diameters with a fixed pulse length of $3 \mathrm{~ns}$. As shown in Fig. 7c, the resistance jumps appear in a similar peaked distributions whatever the size of the device (the noise limit is taken at $2 \%$ according to the previous measurement). Furthermore, the amplitude of the resistance jumps decreases as the diameter of the device increases (Fig.7c, shift towards smaller normalized TMR jumps as the diameter of the device increases). However, if we now recalculate the size of the switching domains taking into account the area of the various devices, we find that the three distributions present the same average value for the diameter of the switching domains of the order of $45 \mathrm{~nm}$ as shown in Fig. $7 \mathrm{~d}$. This tends to confirm the idea that the free layer as a sort of granular behavior with grain size of the order of $45 \mathrm{~nm}$. This phenomenon can be explained with the presence of the IrMn layer in the free layer. Its granular structure and its spin glass-like magnetic state can create, through exchange interaction at the interface with the two ferromagnetic layers, a granular type of magnetic behavior ${ }^{12}$. These grains can switch even around the $\mathrm{P}$ and AP states when the voltage pulses are applied by thermal activation. The temperature reached in the free layer during a voltage pulse can be estimated in a MTJ within a unidimensional model through the expression

$T(t)=T_{0}+\frac{P_{d} A}{K}\left[1-\exp \left(-\frac{t}{\tau}\right)\right]$

where $T_{0}$ is the initial standby temperature (RT), $P_{d}$ the heating power density during the pulse $\left(V^{2} / R A\right), A$ the device area, $\mathrm{K}$ the effective heat conductance between the tunnel barrier and the bottom and top electrodes considered to act as thermal bath at $T_{0}$, $\tau$ the characteristic heating time which depends on the pillar specific heat and thermal conductance and $t$ the pulse length ${ }^{32}$. From earlier studies on thermally assisted MRAM which used relatively similar material stacks ${ }^{29}$, the following statements can be made : For $t<$ $20 \mathrm{~ns}$, the temperature increases rapidly with the pulse length $t$ and almost independently on the device size. For $t>20 \mathrm{~ns}$, the temperature increase tends to slow down due to heat diffusion towards the top and bottom metallic electrodes and gradually the temperature reaches an asymptotic regime which corresponds to a balance between the heat production around the tunnel barrier and the heat flow towards the electrodes. In our experiments using pulses between $1 \mathrm{~ns}$ and $4 \mathrm{~ns}$ long, we are still in a regime where the temperature rise in the MTJ is roughly proportional to the pulse duration. Considering that in thermally assisted MRAM, a temperature rise of about $150^{\circ} \mathrm{C}$ was achieved in about $5 \mathrm{~ns}$ with a heating power of $100 \mathrm{~mW} / \mu \mathrm{m}^{2}$ and taking into account the dependence on the MTJ diameter, we can roughly estimate that in the present junction, a $2 \mathrm{~ns}$ pulse of $0.9 \mathrm{~V}$ can yield an increase of temperature of the order of $40^{\circ} \mathrm{C}$ while a $4 \mathrm{~ns}$ long pulse yields an increase of temperature of about $80^{\circ} \mathrm{C}$. Since the coercivity of the free layer measured in quasistatic mode by VSM tends to vanish at $100^{\circ} \mathrm{C}$, the Joule heating induced by the voltage pulses can indeed trigger the switching of small domains in the free layer.

\section{Conclusions}

In conclusion, we proposed a device based on the angular variation of TMR in an in-plane MTJ. Thanks to the property of isotropic coercivity of a free layer based on a FM/AF/FM structure, we are able to discretely rotate the magnetization by STT and stabilize it at different in-plane angles. We found that in single pulse experiments, we could adjust the relative amplitude of the STT originating from the in-plane analyzer and perpendicular polarizer to limit the angular excursion of the free layer magnetization in a half-plane which is mandatory to achieve a monotonous variation of resistance for each pulse polarity as required for memristor functionality. In the multiple pulses experiments, we demonstrated an average monotonous behavior of the resistance variation with the voltage polarity. An almost continuous variation of resistance could be achieved between a minimum resistance state corresponding to parallel remanent magnetic configuration and a maximum resistance corresponding to antiparallel remanent magnetic configuration. By analyzing few of these levels, resistance variations below $0.1 \%$ in 1 s timescale and below $2 \%$ in 3 minutes were found. For both voltage polarities, random resistance jumps are observed on top of the general 
monotonous trend in resistance variation. These random jumps are associated with thermally activated switching of small domains in the free layer magnetization due to Joule heating occurring during the current pulses. These domains are about $45 \mathrm{~nm}$ in dimension whatever the diameter of the pillar and seems to be associated with a granular structure of the free layer.

The device here described has intrinsically a good down-size scalability since its working principle (based on angular variation of the TMR) is not directly linked to its size. In particular, a reduction in the diameter of such device would first reduce the Joule heating ${ }^{32}$ thanks to additional heat dissipation though the sidewalls of the pillar and, at the same time, bring the device to a more macrospin behavior thus reducing significantly the random contributions occurring during application of the voltage pulses.

Such memristor could be implemented in crossbar architectures to perform the cumulate and multiply function, key in neuromorphic computing ${ }^{33}$.

\section{Acknowledgements}

This work was funded under the ERC Adv grant MAGICAL Nº 669204

\section{References}

1. S. Kim, S.H. Choi, and W. Lu, Comprehensive Physical Model of Dynamic Resistive Switching in an Oxide Memristor, ACSNano, 8, 2369 (2014).

2. A. Chanthbouala et al, A ferroelectric memristor, Nature Mater 11, 860-864 (2012).

3. S. Lequeux et al. A magnetic synapse: multilevel spin-torque memristor with perpendicular anisotropy. Sci. Rep. 6, 31510 (2016).

4. Y.U. Kim, J. Kwon, H.K. Hwang,I. Purnama, and C.Y. You, Multilevel anomalous Hall resistance in a single Hall cross for the applications of neuromorphic device. Sci. Rep. 10, 1285 (2020).

5. M.A. Azam, D. Bhattacharya, D. Querlioz, C. Ross and J. Atulasimha, Voltage control of domain walls in magnetic nanowires for energy-efficient neuromorphic devices. Nanotechnology 31, 145201 (2020).

6. E. Raymenants et al. Chain of magnetic tunnel junctions as a spintronic memristor. J. Appl. Phys. 124, 152116 (2018).

7. V. Ostwal, R. Zand, R. DeMara, and J. Appenzeller, Novel Compound Synapse Using Probabilistic Spin-Orbit-Torque Switching for MTJ-Based Deep Neural Networks. IEEE J. Explor. Solid-State Comput. Devices Circuits 5, 182-187 (2019).

8. S. Fukami, C. Zhang, S. DuttaGupta, A. Kurenkov, and H. Ohno, Magnetization switching by spin-orbit torque in an antiferromagnet-ferromagnet bilayer system. Nat. Mater. 15, 535541 (2016)

9. A. Kurenkov et al, Artificial Neuron and Synapse Realized in an Antiferromagnet/Ferromagnet Heterostructure Using Dynamics of Spin-Orbit Torque Switching. Adv. Mater. 31, 1900636 (2019).

10. J.C. Slonczewski, Conductance and exchange coupling of two ferromagnets separated by a tunneling barrier. Phys. Rev. B 39, 6995-7002 (1989).

11. H. Jaffrès, H. et al. Angular dependence of the tunnel magnetoresistance in transition-metal-based junctions. Phys. Rev. $B$ 64, 064427 (2001).

12. M. Mansueto et al. Realizing an Isotropically Coercive Magnetic Layer for Memristive Applications by Analogy to Dry Friction. Phys. Rev. Appl. 12, 044029 (2019).

13. M. Mansueto et $a$, Isotropically coercive free layer integration in a magnetic tunnel junction for neuromorphic applications. in 2020 IEEE International Memory Workshop (IMW) 1-4 (2020). doi:10.1109/IMW48823.2020.9108129.

14. K. Garello et al, Manufacturable $300 \mathrm{~mm}$ platform solution for Field-Free Switching SOT-MRAM. in 2019 Symposium on VLSI Technology T194-T195 (2019). doi:10.23919/VLSIT.2019.8776537.

15. D. Houssameddine et al, Spin-torque oscillator using a perpendicular polarizer and a planar free layer. Nat. Mater. 6, 447453 (2007)

16. H. Liu et al, Ultrafast switching in magnetic tunnel junction based orthogonal spin transfer devices. Appl. Phys. Lett. 97, 242510 (2010).

17. A. Mejdoubi, B. Lacoste, G. Prenat, and B. Dieny, Macrospin model of precessional spin-transfer-torque switching in planar magnetic tunnel junctions with perpendicular polarizer. Appl. Phys. Lett. 102, 152413 (2013).

18. B. Lacoste, L.D. Buda-Prejbeanu, U. Ebels and B. Dieny, Out-ofplane precession of an in-plane magnetized free layer submitted to the spin-transfer torque of a perpendicular polarizer: An analytical perturbative approach. Phys. Rev. B 88, 054425 (2013).

19 B. Lacoste et al. Modulating spin transfer torque switching dynamics with two orthogonal spin-polarizers by varying the cell aspect ratio. Phys. Rev. B 90, 224404 (2014).

20. B. Lacoste et al. Control of Sub-Nanosecond Precessional Magnetic Switching in STT-MRAM Cells for SRAM Applications. in 2016 IEEE 8th International Memory Workshop (IMW) 1-4 (2016). doi:10.1109/IMW.2016.7495262.

21. J.Z.Sun et al. Spin-torque switching efficiency in CoFeB-MgO based tunnel junctions. Phys. Rev. B 88, 104426 (2013). 
22. M.A. Ruderman and C. Kittel, Indirect Exchange Coupling of Nuclear Magnetic Moments by Conduction Electrons. Phys. Rev. 96, 99-102 (1954).

23. T.A. Kasuya, Theory of Metallic Ferro- and Antiferromagnetism on Zener's Model. Prog. Theor. Phys. 16, 45-57 (1956).

24. K. Yosida, Magnetic Properties of Cu-Mn Alloys. Phys. Rev. 106, 893-898 (1957).

25 W.B. Zeper, F.J.A.M. Greidanus, P.F. Carcia and C.R. Fincher, Perpendicular magnetic anisotropy and magneto-optical Kerr effect of vapor-deposited Co/Pt-layered structures. J. Appl. Phys. 65, 4971-4975 (1989).

26. D. Apalkov, B. Dieny, J. Slaughter, Magnetoresistive Random AccessMemory, Proceedings of the IEEE, 104, 1796 (2016)

27. E.E. Fullerton; J.R. Childress, Spintronics, Magnetoresistive Heads, and the Emergence of the Digital World, Proceedings of the IEEE, 104, 1787 (2016)

28. K.J.Lee, O. Redon and B. Dieny, Analytical investigation of spintransfer dynamics using a perpendicular-to-plane polarizer. Appl Phys Lett 86, 022505 (2005) .

29. D. Spenato, S.P. Pogossian, D.T. Dekadjevi and J.B. Youssef, Study of dynamic properties and magnetic anisotropies of $\mathrm{NiFe} / \mathrm{MnPt}$ in the critical thickness range. J. Phys. Appl. Phys. 40, 3306-3313 (2007).

30. J. McCord, Magnetization Dynamics of Coupled FerromagneticAntiferromagnetic Thin Films. in Advances in Solid State Physics (ed. Haug, R.) vol. 48 157-170 (Springer Berlin Heidelberg, 2009).

31. F. Xu et al, Influence of Thickness on Magnetic Properties and Microwave Characteristics of NiFe/IrMn/NiFe Trilayers. IEEE Trans. Magn. 47, 3486-3489 (2011).

32. I.L. Prejbeanu et al. Thermally assisted MRAM. J. Phys. Condens. Matter 19, 165218 (2007).

33. J. Grollier, D. Querlioz, K. Y. Camsari, K. Everschor-Sitte, S.Fukami and M. D. Stiles, Neuromorphic spintronics. Nat.Elect. 3, 360 (2020). 